EPHOU-13-006

\title{
Renormalization group invariants in neutrino sector
}

\author{
Naoyuki Haba ${ }^{1,2}$ and Ryo Takahashi ${ }^{2}$ \\ ${ }^{1}$ Graduate School of Science and Engineering, Shimane University, \\ Matsue 690-8504, Japan \\ ${ }^{2}$ Department of Physics, Faculty of Science, Hokkaido University, \\ Sapporo 060-0810, Japan
}

\begin{abstract}
We show renormalization group invariants in neutrino sector. These are found from a simple analytical discussion of Majorana mass matrix for light neutrinos. It is shown that the invariants are obtained by taking ratios among elements of the Majorana neutrino mass matrix. The invariance is independent of neutrino mass ordering and a parameterization of mixing matrix for the lepton sector. Parameters in the runnings under renormalization group equations in the neutrino sector are also analyzed.
\end{abstract}




\section{Introduction}

Neutrino physics is one of important clues to look for physics beyond the standard model (SM) because neutrino oscillation experiments established that neutrinos have small masses compared to other SM fermion masses. Moreover recent precision measurements of leptonic mixing angles in the Pontecorvo-Maki-Nakagawa-Sakata (PMNS) matrix [1] showed that $\theta_{12}$ and $\theta_{23}$ are large, and $\theta_{13}$ is small but not zero [2, 3, 4]. As far as the neutrino masses concerned, two mass squared differences are measured. Accordingly, a normal hierarchy (NH), $m_{1}<m_{2}<m_{3}$, or an inverted hierarchy (IH), $m_{3}<m_{1}<m_{2}$, are now allowed where $m_{i}$ are the neutrino mass eigenvalues.

It is, in particular, important to realize the smallness of the neutrino masses. A lot of works have been proposed for the realization of the small masses in the context of the seesaw mechanism [5]. The mechanism implies the presence of new physics (particles) at a high energy regime. In addition to the seesaw mechanism, one may also consider a kind of unified theory, namely a grand unified theory (GUT), and/or a new symmetry, e.g., flavor symmetry, at high energy in order to nicely derive a gauge (group) and Yukawa (masses and generation mixing) structures of the SM at low energy. Therefore, investigations of high energy behavior in the neutrino sector might be important for a clarification of a new physics beyond the SM. Renormalization of group equations (RGEs) for physical parameters are ones of tools to seek a high energy physics. In fact, a large number of works respect with the RGEs of the neutrino sector have been presented (e.g., see [6, 17, 8, 9, 10, 11]).

Supersymmetry (SUSY) is attractive since it can solve some problems in the SM. For instance, the problems are the gauge hierarchy problem and the absence of the dark matter (DM) candidate. Furthermore, the gauge coupling unification can be interestingly realized at high energy scale in the minimal supersymmetric standard model (MSSM). Then, the origin of the tiny neutrino masses, e.g. the heavy right-handed neutrinos, could be naturally embedded into a GUT. In this letter, we investigate a behavior of a coefficient of the Weinberg operator [12, which describes tiny neutrino mass, under the RGEs in the MSSM. 


\section{Analyses under renormalization group equations in neutrino sector}

\subsection{Renormalization group invariants}

We consider effective Yukawa interactions of the lepton sector and the Weinberg operator in the MSSM at low energy such as the electroweak (EW) scale $\Lambda_{\mathrm{EW}}$ in the lepton sector,

$$
\mathcal{L} \supset-y_{e} \bar{L} H_{d} e_{R}+\frac{\kappa}{2}\left(H_{u} L\right)\left(H_{u} L\right)+\text { h.c. },
$$

where $y_{e}, L, e_{R}, H_{d(u)}$, and the second term are a matrix of Yukawa couplings of charged leptons, left-handed lepton doublets, right-handed charged leptons, down(up)-type Higgs, and the Weinberg operator, respectively. A mass matrix for light (active) neutrinos is given by $M_{\nu}=\kappa v_{u}^{2}=\kappa v^{2} \sin ^{2} \beta$ after the Higgs gets a vacuum expectation value (VEV), $v_{u}$ where $\tan \beta \equiv v_{u} / v_{d}$ and $v_{d}$ is a VEV of the down-type Higgs.

The light neutrino mass matrix can also be described by the PMNS matrix and mass eigenvalues of light neutrinos as:

$$
\left(M_{\nu}\right)_{\alpha \beta}=\left(U M_{\nu}^{\operatorname{diag}} U^{T}\right)_{\alpha \beta}=\left(U \cdot \operatorname{Diag}\left\{m_{1}, m_{2}, m_{3}\right\} \cdot U^{T}\right)_{\alpha \beta}=\sum_{i} U_{\alpha i} U_{\beta i} m_{i}
$$

in a diagonal basis of the Yukawa coupling matrix for the charged leptons where $U$ is the PMNS matrix, $M_{\nu}^{\text {diag }}$ is a diagonal matrix, and $\alpha, \beta=e, \mu, \tau$. The light neutrino mass matrix can be described by 3 mixing angles, 3 mass eigenvalues of the neutrinos, and 3 CP-phases (one Dirac and two Majorana phases) if the neutrinos are Majorana particles. To determine these quantities in the neutrino sector by the experiments is one of important goals in studies of neutrino physics. Once one fixes those values at low energy as boundary conditions, one can obtain those values at arbitrary high energy scale by solving corresponding RGEs. To get a structure of neutrino mass matrix with ones of other SM fermion mass matrices strongly motivates to study physics beyond the SM, e.g., GUT and/or the presence of an additional symmetry such as a flavor symmetry.

By solving the RGE for $\kappa$ as:

$$
16 \pi^{2} \frac{d \kappa}{d t}=\bar{\alpha} \kappa+\left(y_{e} y_{e}^{\dagger}\right) \kappa+\kappa\left(y_{e} y_{e}^{\dagger}\right)^{T}
$$

with $t \equiv \ln \mu$ and

$$
\bar{\alpha} \equiv-\frac{6}{5} g_{1}^{2}-6 g_{2}^{2}+6\left(y_{u}^{2}+y_{c}^{2}+y_{t}^{2}\right)
$$

at one-loop level, we can write a Majorana mass matrix of the light neutrinos as $M_{\nu}(\Lambda)=$ $R\left(I M_{\nu}\left(\Lambda_{\mathrm{EW}}\right) I\right)$ at arbitrary high energy scale $\Lambda$ where $\mu$ is a renormalization scale, $g_{i}$ are 
gauge coupling constants, $y_{\alpha_{q}}\left(\alpha_{q}=u, c, t\right)$ are Yukawa couplings of up-type quarks, $R$ is a flavor blind overall factor, and $I$ is defined by $I^{-1} \equiv \operatorname{Diag}\left\{\sqrt{I_{e}}, \sqrt{I_{\mu}}, \sqrt{I_{\tau}}\right\}$ [7, 8, 10]. $I_{\alpha}$ denote quantum corrections for the Yukawa couplings as $I_{\alpha} \equiv \exp \left[\frac{1}{8 \pi^{2}} \int_{t_{\Lambda}}^{t_{\mathrm{EW}}} d t y_{\alpha}^{2}\right]$ with $t_{\Lambda} \equiv \ln \Lambda$ and $t_{\mathrm{EW}} \equiv \ln \Lambda_{\mathrm{EW}}$. A dominant effect of the quantum corrections comes from the coupling $y_{\tau}$, and thus we introduce small parameters defined as $\epsilon_{e} \equiv \sqrt{\frac{I_{\tau}}{I_{e}}}-1$ and $\epsilon_{\mu} \equiv \sqrt{\frac{I_{\tau}}{I_{\mu}}}-1$. Since $\epsilon_{e}$ and $\epsilon_{\mu}$ are numerically well approximated as $\epsilon_{e}=\epsilon_{\mu}$, we take $\epsilon=\epsilon_{e}=\epsilon_{\mu}$ in the following discussions. Then, the neutrino mass matrix at arbitrary high energy scale can be well approximated by

$$
M_{\nu}(\Lambda)=r\left(\begin{array}{ccc}
\left(M_{\nu}\left(\Lambda_{\mathrm{EW}}\right)\right)_{e e} & \left(M_{\nu}\left(\Lambda_{\mathrm{EW}}\right)\right)_{e \mu} & \left(M_{\nu}\left(\Lambda_{\mathrm{EW}}\right)\right)_{e \tau}(1+\epsilon) \\
\left(M_{\nu}\left(\Lambda_{\mathrm{EW}}\right)\right)_{e \mu} & \left(M_{\nu}\left(\Lambda_{\mathrm{EW}}\right)\right)_{\mu \mu} & \left(M_{\nu}\left(\Lambda_{\mathrm{EW}}\right)\right)_{\mu \tau}(1+\epsilon) \\
\left(M_{\nu}\left(\Lambda_{\mathrm{EW}}\right)\right)_{e \tau}(1+\epsilon) & \left(M_{\nu}\left(\Lambda_{\mathrm{EW}}\right)\right)_{\mu \tau}(1+\epsilon) & \left(M_{\nu}\left(\Lambda_{\mathrm{EW}}\right)\right)_{\tau \tau}(1+\epsilon)^{2}
\end{array}\right)
$$

where $r \equiv R / I_{e}$.

Let us consider renormalization group invariants for the neutrino parameters. First, it has been pointed out that argument of all the matrix elements of $\kappa$ do not evolve under the RGEs [9]. The reason is as follows: the RGE of $\kappa$ can be rewritten as,

$$
\frac{d}{d t} \ln \kappa_{i j}=\frac{d}{d t} \ln \left|\kappa_{i j}\right|+i \frac{d}{d t} \phi_{i j}=\gamma_{i}+\gamma_{j}+\gamma_{H},
$$

with a notation $\kappa_{i j} \equiv\left|\kappa_{i j}\right| e^{i \phi_{i j}}$ where $\gamma_{i}$ and $\gamma_{H}$ are anomalous dimensions, which are real, for the left-handed lepton doublets and the up-type Higgs defined by those wave function renormalizations, respectively. Therefore, this leads to $d \phi_{i j} / d t=0$. Are there any other renormalization group invariants in addition to CP-phases in $\kappa$ ?

When one compare (5) with (2), one can interestingly find 4 renormalization group invariants as, 1

$$
\begin{aligned}
& \frac{\left(M_{\nu}(\Lambda)\right)_{e e}}{\left(M_{\nu}(\Lambda)\right)_{e \mu}}=\frac{\left(M_{\nu}\left(\Lambda_{\mathrm{EW}}\right)\right)_{e e}}{\left(M_{\nu}\left(\Lambda_{\mathrm{EW}}\right)\right)_{e \mu}}=\frac{\sum_{i} U_{e i}^{2}\left(\Lambda_{\mathrm{EW}}\right) m_{i}\left(\Lambda_{\mathrm{EW}}\right)}{\sum_{i} U_{e i}\left(\Lambda_{\mathrm{EW}}\right) U_{\mu i}\left(\Lambda_{\mathrm{EW}}\right) m_{i}\left(\Lambda_{\mathrm{EW}}\right)}=\frac{m_{e e}}{m_{e \mu}} \\
& \frac{\left(M_{\nu}(\Lambda)\right)_{e e}}{\left(M_{\nu}(\Lambda)\right)_{\mu \mu}}=\frac{\left(M_{\nu}\left(\Lambda_{\mathrm{EW}}\right)\right)_{e e}}{\left(M_{\nu}\left(\Lambda_{\mathrm{EW}}\right)\right)_{\mu \mu}}=\frac{\sum_{i} U_{e i}^{2}\left(\Lambda_{\mathrm{EW}}\right) m_{i}\left(\Lambda_{\mathrm{EW}}\right)}{\sum_{i} U_{\mu i}^{2}\left(\Lambda_{\mathrm{EW}}\right) m_{i}\left(\Lambda_{\mathrm{EW}}\right)}=\frac{m_{e e}}{m_{\mu \mu}} \\
& \frac{\left(M_{\nu}(\Lambda)\right)_{e \tau}}{\left(M_{\nu}(\Lambda)\right)_{\mu \tau}}=\frac{\left(M_{\nu}\left(\Lambda_{\mathrm{EW}}\right)\right)_{e \tau}}{\left(M_{\nu}\left(\Lambda_{\mathrm{EW}}\right)\right)_{\mu \tau}}=\frac{\sum_{i} U_{e i}\left(\Lambda_{\mathrm{EW}}\right) U_{\tau i}\left(\Lambda_{\mathrm{EW}}\right) m_{i}\left(\Lambda_{\mathrm{EW}}\right)}{\sum_{i} U_{\mu i}\left(\Lambda_{\mathrm{EW}}\right) U_{\tau i}\left(\Lambda_{\mathrm{EW}}\right) m_{i}\left(\Lambda_{\mathrm{EW}}\right)}=\frac{m_{e \tau}}{m_{\mu \tau}} \\
& \frac{\left(M_{\nu}(\Lambda)\right)_{e \tau}^{2}}{\left(M_{\nu}(\Lambda)\right)_{e e}\left(M_{\nu}(\Lambda)\right)_{\tau \tau}}=\frac{\left(M_{\nu}\left(\Lambda_{\mathrm{EW}}\right)\right)_{e \tau}^{2}}{\left(M_{\nu}\left(\Lambda_{\mathrm{EW}}\right)\right)_{e e}\left(M_{\nu}\left(\Lambda_{\mathrm{EW}}\right)\right)_{\tau \tau}} \\
& =\frac{\left(\sum_{i} U_{e i}\left(\Lambda_{\mathrm{EW}}\right) U_{\tau i}\left(\Lambda_{\mathrm{EW}}\right) m_{i}\left(\Lambda_{\mathrm{EW}}\right)\right)^{2}}{\left(\sum_{i} U_{e i}^{2}\left(\Lambda_{\mathrm{EW}}\right) m_{i}\left(\Lambda_{\mathrm{EW}}\right)\right)\left(\sum_{i} U_{\tau i}^{2}\left(\Lambda_{\mathrm{EW}}\right) m_{i}\left(\Lambda_{\mathrm{EW}}\right)\right)}=\frac{m_{e \tau}^{2}}{m_{e e} m_{\tau \tau}} .
\end{aligned}
$$

We show values of these invariants in Figures 1 and 2 . Figures 1 and 2 are cases that all CP-phases are relatively small $(0 \leq(\delta, \alpha, \beta)<2 \pi / 3)$ and large $(4 \pi / 3 \leq(\delta, \alpha, \beta)<2 \pi)$,

\footnotetext{
${ }^{1}$ See also $[13$ for a property of a strong scaling ansatz under the RGEs.
} 
respectively, where $\delta, \alpha$ and $\beta$ are a Dirac and two Majorana phases, respectively. The calculations are given within $3 \sigma$ range for experimentally determined values of the neutrino parameters [4] as:

$$
\begin{aligned}
& 0.27 \leq \sin ^{2} \theta_{12} \leq 0.34, \quad 0.34 \leq \sin ^{2} \theta_{23} \leq 0.67, \quad 0.016 \leq \sin ^{2} \theta_{13} \leq 0.030 \\
& 7.00 \times 10^{-5} \mathrm{eV}^{2} \leq \Delta m_{21}^{2} \leq 8.09 \times 10^{-5} \mathrm{eV}^{2} \\
& 2.27 \times 10^{-3} \mathrm{eV}^{2} \leq \Delta m_{31}^{2} \leq 2.69 \times 10^{-3} \mathrm{eV}^{2} \\
& -2.65 \times 10^{-3} \mathrm{eV}^{2} \leq \Delta m_{32}^{2} \leq-2.24 \times 10^{-3} \mathrm{eV}^{2}
\end{aligned}
$$

The horizontal axes in all figures of Figures 1 and 2 are the sum of the three light neutrino masses $\sum m_{\nu}$ which is constrained by the first cosmological result based on Planck measurements of the cosmic microwave background combined with other cosmological data as $\sum m_{\nu}<0.23 \mathrm{eV}$ at $95 \% \mathrm{CL}$ [14].2

We find from Figures 1 and 2 that

$$
\begin{aligned}
& 5 \times 10^{-2} \lesssim\left|\frac{m_{e e}}{m_{e \mu}}\right| \lesssim 2 \times 10^{2}, \quad 10^{-3} \lesssim\left|\frac{m_{e e}}{m_{\mu \mu}}\right| \lesssim 3 \times 10^{2}, \\
& 2 \times 10^{-3} \lesssim\left|\frac{m_{e \tau}}{m_{\mu \tau}}\right| \lesssim 5, \quad 8 \times 10^{-6} \lesssim\left|\frac{m_{e \tau}^{2}}{m_{e e} m_{\mu \tau}}\right| \lesssim 10^{2}
\end{aligned}
$$

for the $\mathrm{NH}$ with $0 \leq(\delta, \alpha, \beta)<2 \pi / 3$, and

$$
\begin{aligned}
& 0.3 \lesssim\left|\frac{m_{e e}}{m_{e \mu}}\right| \lesssim 6 \times 10^{2}, \quad 0.3 \lesssim\left|\frac{m_{e e}}{m_{\mu \mu}}\right| \lesssim 3 \times 10^{2} \\
& 1.5 \times 10^{-3} \lesssim\left|\frac{m_{e \tau}}{m_{\mu \tau}}\right| \lesssim 7, \quad 2 \times 10^{-6} \lesssim\left|\frac{m_{e \tau}^{2}}{m_{e e} m_{\mu \tau}}\right| \lesssim 70,
\end{aligned}
$$

for the $\mathrm{IH}$ with $0 \leq(\delta, \alpha, \beta)<2 \pi / 3$, and

$$
\begin{aligned}
& 10^{-2} \lesssim\left|\frac{m_{e e}}{m_{e \mu}}\right| \lesssim 3 \times 10^{2}, \quad 3 \times 10^{-3} \lesssim\left|\frac{m_{e e}}{m_{\mu \mu}}\right| \lesssim 3 \times 10^{2}, \\
& 9 \times 10^{-4} \lesssim\left|\frac{m_{e \tau}}{m_{\mu \tau}}\right| \lesssim 5, \quad 8 \times 10^{-7} \lesssim\left|\frac{m_{e \tau}^{2}}{m_{e e} m_{\mu \tau}}\right| \lesssim 10^{2},
\end{aligned}
$$

for the $\mathrm{NH}$ with $4 \pi / 3 \leq(\delta, \alpha, \beta)<2 \pi$, and

$$
\begin{aligned}
& 0.3 \lesssim\left|\frac{m_{e e}}{m_{e \mu}}\right| \lesssim 5 \times 10^{2}, \quad 0.3 \lesssim\left|\frac{m_{e e}}{m_{\mu \mu}}\right| \lesssim 2 \times 10^{2}, \\
& 10^{-3} \lesssim\left|\frac{m_{e \tau}}{m_{\mu \tau}}\right| \lesssim 8, \quad 10^{-6} \lesssim\left|\frac{m_{e \tau}^{2}}{m_{e e} m_{\mu \tau}}\right| \lesssim 6
\end{aligned}
$$

\footnotetext{
${ }^{2}$ See also [15] for constraints on neutrino mass ordering and degeneracy from the Planck and neutrino-less double beta decay.
} 

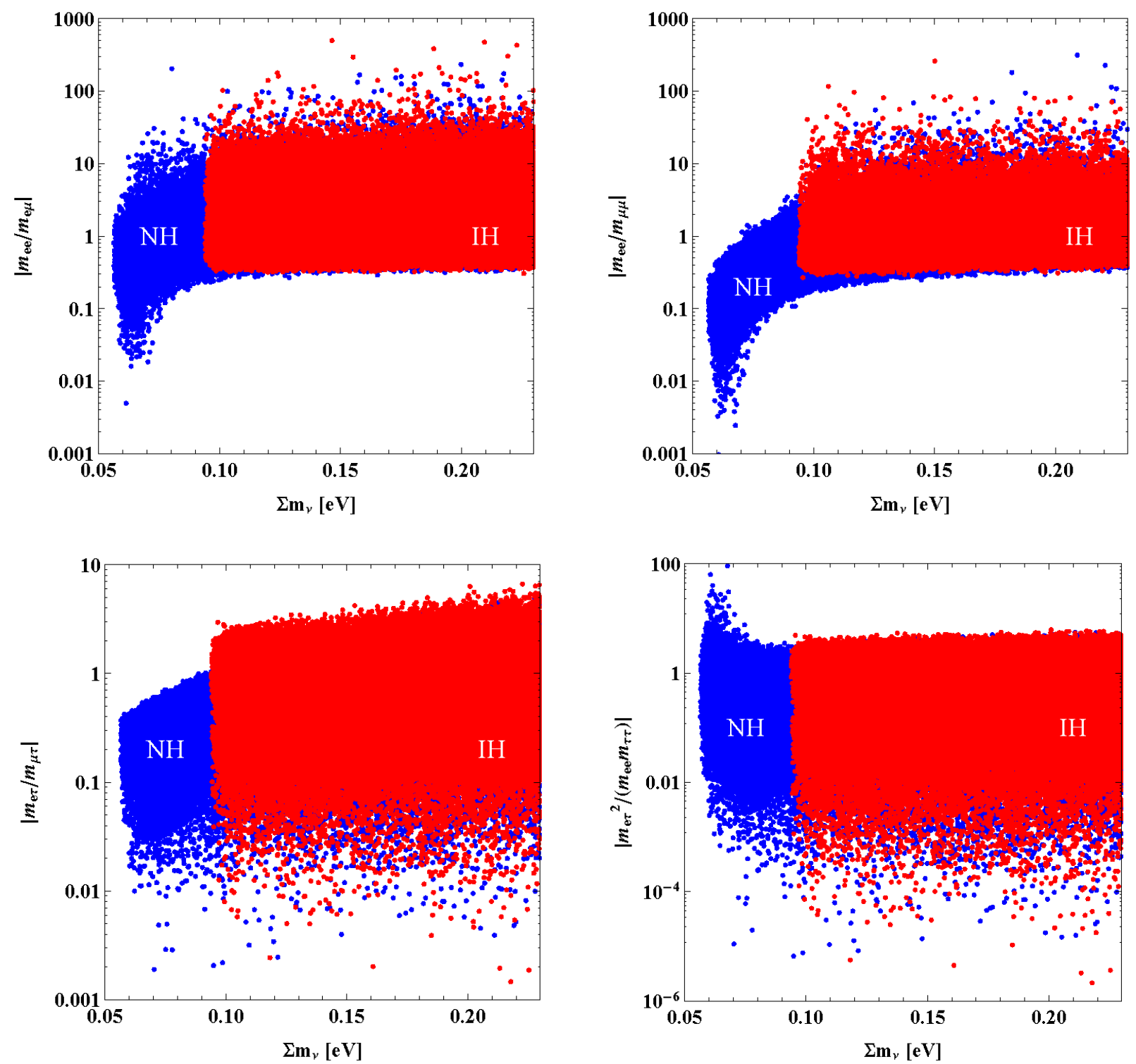

Figure 1: Values of the renormalization invariants vs the sum of the neutrino masses in the $0 \leq(\delta, \alpha, \beta)<2 \pi / 3$ case. 

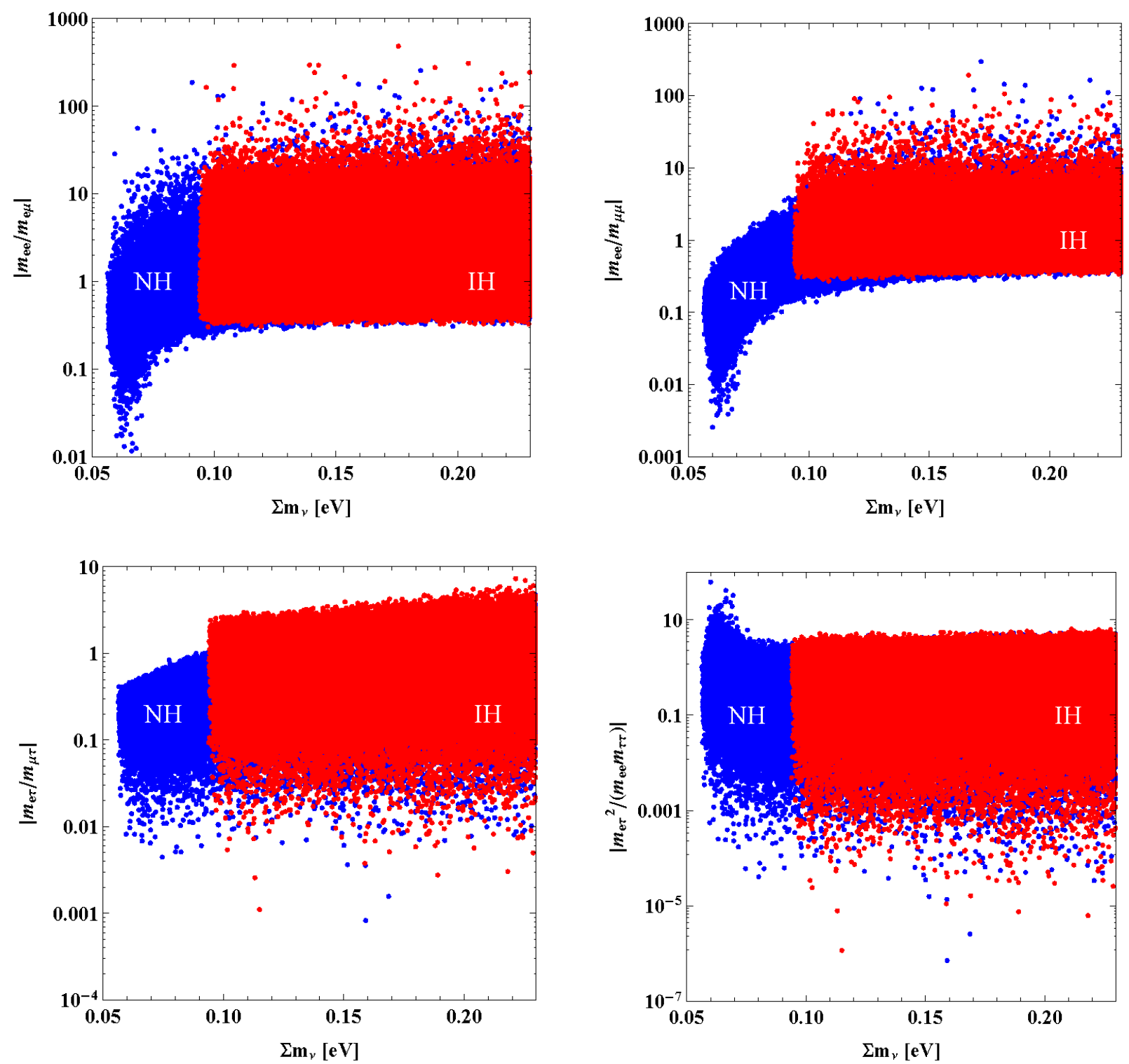

Figure 2: Values of the renormalization invariants vs the sum of the neutrino masses in the $4 \pi / 3 \leq(\delta, \alpha, \beta)<2 \pi$. 
for the IH with $4 \pi / 3 \leq(\delta, \alpha, \beta)<2 \pi$. We can also consider other combination of sizes of CP phases. The results of other possible cases are summarized in Tables 1 6 in Appendix.

The invariance is independent of neutrino mass ordering and a parameterization of the PMNS matrix. The other quantities by using these invariants are also RGE invariants. In a general Majorana mass matrix for the 3 generations of the light neutrinos, there are 9 degrees of freedom, which are described by 3 mixing angles, 3 masses and $3 \mathrm{CP}$-phases. Regarding the number of the RGE invariants, $3 \mathrm{CP}$ phases [9] and the above 4 combinations among matrix elements in (5) are the independent RGE invariants. Therefore, the remaining 2 quantities are not RGE invariants. These are the overall factor $r$ and the small parameter $\epsilon$. In the next subsection, we show runnings of these parameters.

\subsection{Runnings of $r$ and $\epsilon$}

We show runnings of $r$ and $\epsilon$ in this subsection. Clearly, the running of $r$ does not affects on the mixing angles while the one of $\epsilon$ affects on them. The running of $r$ is determined by the solving the RGE including only flavor blind effects as:

$$
16 \pi^{2} \frac{d \kappa_{33}}{d t}=\bar{\alpha} \kappa_{33}+\left[\left(y_{e} y_{e}^{\dagger}\right) \kappa+\kappa\left(y_{e} y_{e}^{\dagger}\right)^{T}\right]_{33}
$$

with a relation

$$
r(\mu)=\frac{\kappa_{33}(\mu)}{\kappa_{33}\left(\Lambda_{\mathrm{EW}}\right)(1+\epsilon)^{2}}=\frac{\left(M_{\nu}(\mu)\right)_{\tau \tau}}{\left(M_{\nu}\left(\Lambda_{\mathrm{EW}}\right)\right)_{\tau \tau}(1+\epsilon)^{2}} .
$$

The running of $\epsilon$ is determined by RGEs for the Yukawa couplings of charged leptons as

$$
16 \pi^{2} \frac{d y_{\alpha}}{d t}=y_{\alpha}\left(4 y_{\alpha}^{*} y_{\alpha}+3 y_{b}^{*} y_{b}-3 g_{2}^{2}-\frac{9}{5} g_{1}^{2}\right)
$$

for $\alpha=\mu, \tau$, where $y_{b}$ is a Yukawa coupling of bottom quark. The runnings of $r$ and $\epsilon$ are given in Fig. 3. In the calculations, a SUSY threshold is taken at $10^{3} \mathrm{GeV}$ and the computations are shown within $10^{3} \mathrm{GeV} \leq \mu \leq 10^{14} \mathrm{GeV} 3$ Since the neutrino Yukawa coupling exceeds 1 at higher energy scale than $10^{14} \mathrm{GeV}$ in the seesaw mechanism, we consider the energy scale lower than $10^{14} \mathrm{GeV}$.

Note that these runnings are independent of the neutrino mass spectra including mass ordering. For the running of the overall factor $r$ in Fig. 3 (a), a contribution from the top Yukawa coupling to $\bar{\alpha}$ in (44) is dominant up to an energy scale lower than $\mu \sim \mathcal{O}\left(10^{9-10}\right) \mathrm{GeV}$ from low energy. On the other hand, contributions from the gauge couplings to $\bar{\alpha}$ become

\footnotetext{
${ }^{3}$ We neglect running effects between the EW scale and the SUSY scale, for simplicity. Therefore, $r=1$ and $\epsilon=0$ are taken at $10^{3} \mathrm{GeV}$ as boundary conditions for the RGE.
} 

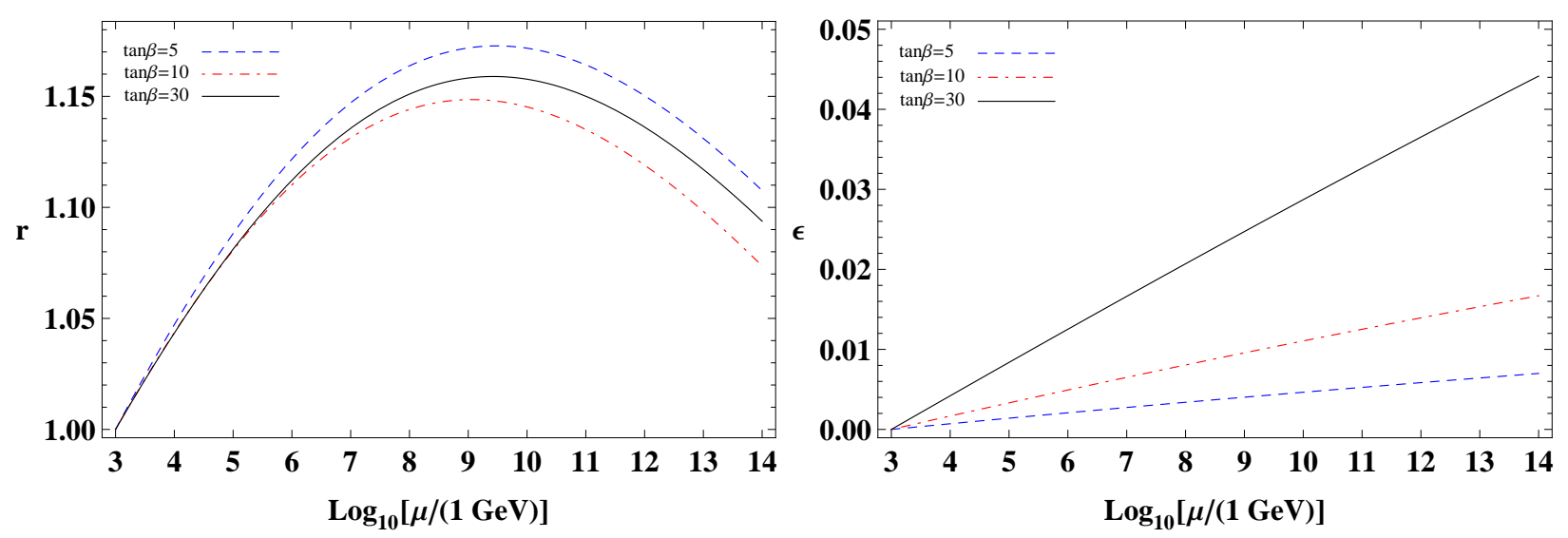

Figure 3: Runnings of the overall factor and small parameter $\epsilon$ in the neutrino mass matrix.

dominant at a higher energy scale than $\mu \sim \mathcal{O}\left(10^{9-10}\right) \mathrm{GeV}$. Therefore, there are peaks around $\mu \sim \mathcal{O}\left(10^{9-10}\right) \mathrm{GeV}$ in the runnings of the overall factor for all cases of $\tan \beta$. As a result, the values of $r$ can be in ranges of $1.00 \leq r \lesssim(1.18,1.15,1.16)$ in $\tan \beta=(5,10,30)$ cases for $10^{3} \mathrm{GeV} \leq \mu \leq 10^{14} \mathrm{GeV}$, respectively. For $\tan \beta=10$ case, the top Yukawa coupling is smaller than that of $\tan \beta=5$ case up to a high energy scale as $\mu \simeq 10^{14} \mathrm{GeV}$. Thus, the curve of running for $\tan \beta=10$ case is lower than one of $\tan \beta=5$ case and the position of the peak appears at smaller $r$ and lower $\mu$ compared to $\tan \beta=5$ case. In particular, the position of the peak for $\tan \beta=10$ case appears at the smallest $r$ and $\mu$ among all cases. On the other hand, the top Yukawa coupling for $\tan \beta=30$ case is the smallest among all cases of $\tan \beta$ at $10^{3} \mathrm{GeV}$ as shown in Fig. 4 (a), but it becomes the largest at higher energy scale because of a non-negligible contribution from a large bottom Yukawa coupling to a running of the top Yukawa as shown in Fig. 目 (b). The values of $r=1$ return at $\mu \simeq 2.20 \times 10^{17}, 1.11 \times 10^{16}$, and $2.82 \times 10^{15} \mathrm{GeV}$ in $\tan \beta=5,10$, and 30 cases, respectively. In the cases, values of the matrix elements such as $\left(M_{\nu}(\mu)\right)_{e e},\left(M_{\nu}(\mu)\right)_{e \mu}$, and $\left(M_{\nu}(\mu)\right)_{\mu \mu}$ are the same as ones at the low energy scale. However, the neutrino Yukawa coupling exceeds 1 at these energy scales so that we do not consider anymore $r=1$ returning point at the high energy. As far as the running of small parameter $\epsilon$ concerned, the values are smaller than $7.00 \times 10^{-3}, 1.39 \times 10^{-2}$, and $4.42 \times 10^{-2}$ for $\tan \beta=5,10$, and 30 , respectively when one considers the renormalization scale up to $10^{14} \mathrm{GeV}$. By using the values of $r$ and $\epsilon$ shown in Fig. 3 with experimentally observed values of the neutrino parameters, one can determine a values of matrix elements in (5) at an arbitrary high energy scale. 
(a)

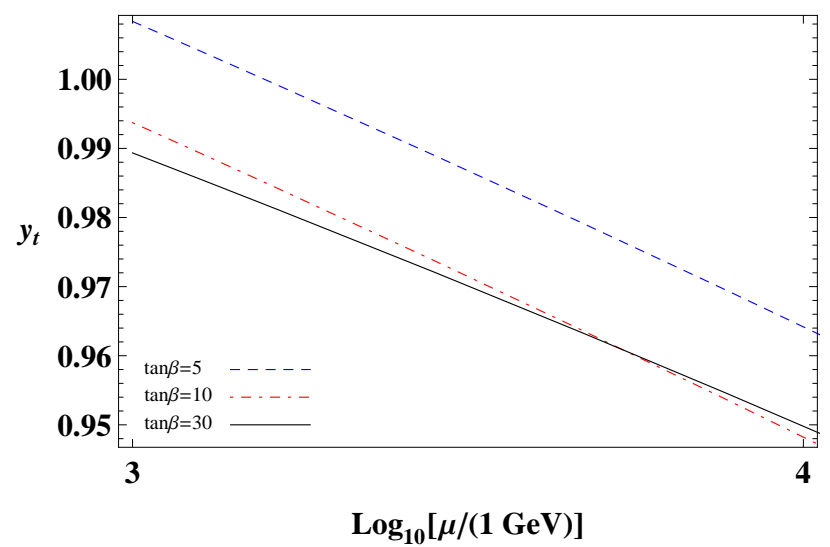

(b)

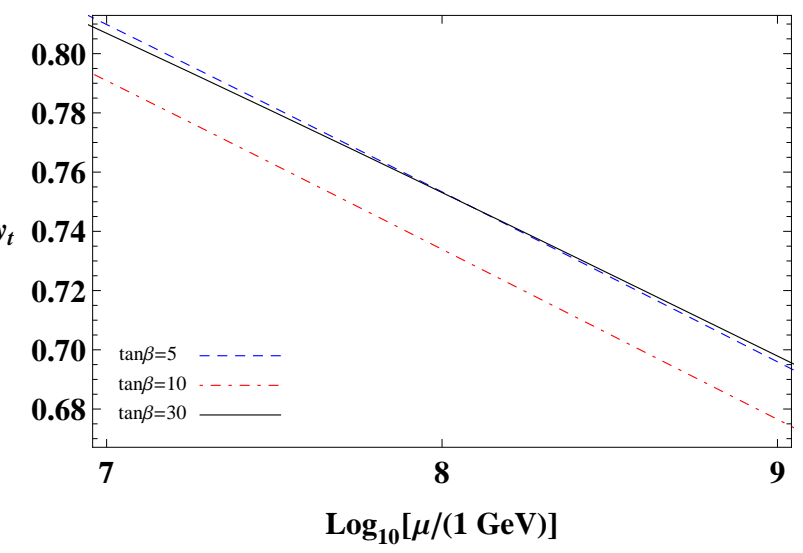

Figure 4: Runnings of the top Yukawa coupling.

\section{Summary}

We investigated a behavior of the coefficient of the Weinberg operator, which describes tiny neutrino masses, under the RGEs at arbitrary high energy regime because the neutrinos might be a key for a study of new physics beyond the SM. A simple analytical discussion clarified that there are several renormalization invariants, which are described by ratios among elements of the Majorana mass matrix of the light neutrinos as $m_{e e} / m_{e \mu}, m_{e e} / m_{\mu \mu}$, $m_{e \tau} / m_{\mu \tau}$, and $m_{e \tau}^{2} /\left(m_{e e} m_{\tau \tau}\right)$. The invariance is independent of neutrino mass ordering and a parameterization of the PMNS matrix. The values are within the ranges of $10^{-3} \lesssim$ $\left|m_{e e} / m_{e \mu}\right| \lesssim 3 \times 10^{3}, 10^{-3} \lesssim\left|m_{e e} / m_{\mu \mu}\right| \lesssim 6 \times 10^{2}, 1.2 \times 10^{-4} \lesssim\left|m_{e \tau} / m_{\mu \tau}\right| \lesssim 6$, and $8 \times 10^{-7} \lesssim\left|m_{e \tau}^{2} /\left(m_{e e} m_{\mu \tau}\right)\right| \lesssim 10^{2}$ for the $\mathrm{NH}$ and $0.2 \lesssim\left|m_{e e} / m_{e \mu}\right| \lesssim 1.8 \times 10^{3}, 0.2 \lesssim$ $\left|m_{e e} / m_{\mu \mu}\right| \lesssim 8 \times 10^{2}, 10^{-3} \lesssim\left|m_{e \tau} / m_{\mu \tau}\right| \lesssim 8$, and $8 \times 10^{-7} \lesssim\left|m_{e \tau}^{2} /\left(m_{e e} m_{\mu \tau}\right)\right| \lesssim 70$ for the $\mathrm{IH}$.

Next we estimated the runnings of the overall factor $r$ and small parameter $\epsilon$ under the corresponding RGEs. Once one determines these parameters at arbitrary high energy scale, one can obtain the values of neutrino mass matrix elements at the high energy. As a result, we found that the values of $r$ can be in ranges of $1 \leq r \lesssim(1.18,1.15,1.16)$ in $\tan \beta=(5,10,30)$ cases for $10^{3} \mathrm{GeV} \leq \mu \leq 10^{14} \mathrm{GeV}$, respectively. As far as the running of small parameter $\epsilon$ concerned, the values are smaller than $7.00 \times 10^{-3}, 1.39 \times 10^{-2}$, and $4.42 \times 10^{-2}$ for $\tan \beta=5,10$, and 30, respectively when one considers the renormalization scale up to $10^{14} \mathrm{GeV}$. 


\section{Acknowledgement}

This work is partially supported by Scientific Grant by Ministry of Education and Science, Nos. 00293803, 20244028, 21244036, 23340070, and by the SUHARA Memorial Foundation. The work of R.T. is supported by Research Fellowships of the Japan Society for the Promotion of Science for Young Scientists.

\section{Appendix}

We show the numerical results of renormalization group invariants for other possible combinations of the CP phases in the following Tables 마.6.

\section{References}

[1] Z. Maki, M. Nakagawa and S. Sakata, Prog. Theor. Phys. 28 (1962) 870; B. Pontecorvo, Sov. Phys. JETP 26 (1968) 984 [Zh. Eksp. Teor. Fiz. 53 (1967) 1717].

[2] K. Abe et al. [T2K Collaboration], Phys. Rev. Lett. 107 (2011) 041801 arXiv:1106.2822 [hep-ex]]; P. Adamson et al. [MINOS Collaboration], Phys. Rev. Lett. 107 (2011) 181802 arXiv:1108.0015 [hep-ex]]; Y. Abe et al. [DOUBLE-CHOOZ Collaboration], Phys. Rev. Lett. 108, 131801 (2012) [arXiv:1112.6353 [hep-ex]]; F. P. An et al. [DAYABAY Collaboration], arXiv:1203.1669 [hep-ex]; J. K. Ahn et al. [RENO collaboration], arXiv:1204.0626 [hep-ex].

[3] M. Tortola, J. W. F. Valle, and D. Vanegas, arXiv:1205.4018 [hep-ph]; G. L. Fogli, E. Lisi, A. Marrone, D. Montanino, A. Palazzo and A. M. Rotunno, Phys. Rev. D 86 (2012) 013012 arXiv:1205.5254 [hep-ph]].

[4] M. C. Gonzalez-Garcia, M. Maltoni, J. Salvado and T. Schwetz, JHEP 1212 (2012) 123 arXiv:1209.3023 [hep-ph]].

[5] P. Minkowski, Phys. Lett. B67 (1977) 421; T. Yanagida, in Proceedings of the Workshop on Unified Theories and Baryon Number in the Universe, eds. O. Sawada and A. Sugamoto (KEK report 79-18, 1979); M. Gell-Mann, P. Ramond and R. Slansky, in Supergravity, eds. P. van Nieuwenhuizen and D.Z. Freedman (North Holland, Amsterdam, 1979); R. N. Mohapatra and G. Senjanovic, Phys. Rev. Lett. 44 (1980) 912; J. Schechter and J. W. F. Valle, Phys. Rev. D 22 (1980) 2227; Phys. Rev. D 25 (1982) 774 . 


\begin{tabular}{|c|c|}
\hline \multicolumn{2}{|c|}{$0 \leq(\delta, \alpha)<2 \pi / 3$ and $2 \pi / 3 \leq \beta<4 \pi / 3$} \\
\hline \hline $3 \times 10^{-3} \lesssim\left|m_{e e} / m_{e \mu}\right| \lesssim 3 \times 10^{2}$ & $1.5 \times 10^{-3} \lesssim\left|m_{e e} / m_{\mu \mu}\right| \lesssim 2 \times 10^{2}$ \\
\hline $2 \times 10^{-3} \lesssim\left|m_{e \tau} / m_{\mu \tau}\right| \lesssim 6$ & $10^{-5} \lesssim\left|m_{e \tau}^{2} /\left(m_{e e} m_{\mu \tau}\right)\right| \lesssim 10^{2}$ \\
\hline
\end{tabular}

\begin{tabular}{|c|c|}
\hline \multicolumn{3}{|c|}{$0 \leq(\delta, \alpha)<2 \pi / 3$ and $4 \pi / 3 \leq \beta<2 \pi$} \\
\hline \hline $2 \times 10^{-3} \lesssim\left|m_{e e} / m_{e \mu}\right| \lesssim 3 \times 10^{2}$ & $10^{-3} \lesssim\left|m_{e e} / m_{\mu \mu}\right| \lesssim 1.5 \times 10^{2}$ \\
\hline $5 \times 10^{-4} \lesssim\left|m_{e \tau} / m_{\mu \tau}\right| \lesssim 4$ & $8 \times 10^{-7} \lesssim\left|m_{e \tau}^{2} /\left(m_{e e} m_{\mu \tau}\right)\right| \lesssim 50$ \\
\hline
\end{tabular}

\begin{tabular}{|c|c|}
\hline \multicolumn{2}{|c|}{$0 \leq(\delta, \beta)<2 \pi / 3$ and $2 \pi / 3 \leq \alpha<4 \pi / 3$} \\
\hline \hline $0.1 \lesssim\left|m_{e e} / m_{e \mu}\right| \lesssim 5 \times 10^{2}$ & $2 \times 10^{-2} \lesssim\left|m_{e e} / m_{\mu \mu}\right| \lesssim 3 \times 10^{2}$ \\
\hline $6 \times 10^{-4} \lesssim\left|m_{e \tau} / m_{\mu \tau}\right| \lesssim 5$ & $10^{-6} \lesssim\left|m_{e \tau}^{2} /\left(m_{e e} m_{\mu \tau}\right)\right| \lesssim 5$ \\
\hline
\end{tabular}

\begin{tabular}{|c|c|}
\hline \multicolumn{2}{|c|}{$0 \leq \delta<2 \pi / 3$ and $2 \pi / 3 \leq(\alpha, \beta)<4 \pi / 3$} \\
\hline \hline $0.1 \lesssim\left|m_{e e} / m_{e \mu}\right| \lesssim 4 \times 10^{2}$ & $3 \times 10^{-2} \lesssim\left|m_{e e} / m_{\mu \mu}\right| \lesssim 1.1 \times 10^{2}$ \\
\hline $1.2 \times 10^{-4} \lesssim\left|m_{e \tau} / m_{\mu \tau}\right| \lesssim 6$ & $3 \times 10^{-8} \lesssim\left|m_{e \tau}^{2} /\left(m_{e e} m_{\mu \tau}\right)\right| \lesssim 5$ \\
\hline
\end{tabular}

\begin{tabular}{|c|c|}
\hline \multicolumn{2}{|c|}{$0 \leq \delta<2 \pi / 3,2 \pi / 3 \leq \alpha<4 \pi / 3$ and $4 \pi / 3 \leq \beta<2 \pi$} \\
\hline \hline $8 \times 10^{-2} \lesssim\left|m_{e e} / m_{e \mu}\right| \lesssim 3 \times 10^{3}$ & $2 \times 10^{-2} \lesssim\left|m_{e e} / m_{\mu \mu}\right| \lesssim 1.5 \times 10^{2}$ \\
\hline $5 \times 10^{-4} \lesssim\left|m_{e \tau} / m_{\mu \tau}\right| \lesssim 3.5$ & $10^{-6} \lesssim\left|m_{e \tau}^{2} /\left(m_{e e} m_{\mu \tau}\right)\right| \lesssim 6$ \\
\hline
\end{tabular}

\begin{tabular}{|c|c|}
\hline \multicolumn{3}{|c|}{$0 \leq(\delta, \beta)<2 \pi / 3$ and $4 \pi / 3 \leq \alpha<2 \pi$} \\
\hline \hline $4 \times 10^{-3} \lesssim\left|m_{e e} / m_{e \mu}\right| \lesssim 9 \times 10^{2}$ & $1.2 \times 10^{-3} \lesssim\left|m_{e e} / m_{\mu \mu}\right| \lesssim 2 \times 10^{2}$ \\
\hline $2 \times 10^{-3} \lesssim\left|m_{e \tau} / m_{\mu \tau}\right| \lesssim 3.5$ & $10^{-5} \lesssim\left|m_{e \tau}^{2} /\left(m_{e e} m_{\mu \tau}\right)\right| \lesssim 10^{2}$ \\
\hline
\end{tabular}

\begin{tabular}{|c|c|}
\hline \multicolumn{2}{|c|}{$0 \leq \delta<2 \pi / 3,4 \pi / 3 \leq \alpha<2 \pi$ and $2 \pi / 3 \leq \beta<4 \pi / 3$} \\
\hline \hline $4 \times 10^{-3} \lesssim\left|m_{e e} / m_{e \mu}\right| \lesssim 2.5 \times 10^{2}$ & $1.2 \times 10^{-3} \lesssim\left|m_{e e} / m_{\mu \mu}\right| \lesssim 2 \times 10^{2}$ \\
\hline $2 \times 10^{-3} \lesssim\left|m_{e \tau} / m_{\mu \tau}\right| \lesssim 3.2$ & $10^{-5} \lesssim\left|m_{e \tau}^{2} /\left(m_{e e} m_{\mu \tau}\right)\right| \lesssim 80$ \\
\hline
\end{tabular}

\begin{tabular}{|c|c|}
\hline \multicolumn{2}{|c|}{$0 \leq \delta<2 \pi / 3$ and $4 \pi / 3 \leq(\alpha, \beta)<2 \pi$} \\
\hline \hline $6 \times 10^{-3} \lesssim\left|m_{e e} / m_{e \mu}\right| \lesssim 7 \times 10^{2}$ & $2 \times 10^{-3} \lesssim\left|m_{e e} / m_{\mu \mu}\right| \lesssim 2 \times 10^{2}$ \\
\hline $6 \times 10^{-4} \lesssim\left|m_{e \tau} / m_{\mu \tau}\right| \lesssim 3$ & $5 \times 10^{-5} \lesssim\left|m_{e \tau}^{2} /\left(m_{e e} m_{\mu \tau}\right)\right| \lesssim 40$ \\
\hline
\end{tabular}

Table 1: Values of the renormalization invariants for the NH. 


\begin{tabular}{|c|c|}
\hline \multicolumn{2}{|c|}{$2 \pi / 3 \leq \delta<4 \pi / 3$ and $0 \leq(\alpha, \beta)<2 \pi / 3$} \\
\hline \hline $8 \times 10^{-3} \lesssim\left|m_{e e} / m_{e \mu}\right| \lesssim 1.3 \times 10^{3}$ & $2 \times 10^{-3} \lesssim\left|m_{e e} / m_{\mu \mu}\right| \lesssim 3 \times 10^{2}$ \\
\hline $1.8 \times 10^{-3} \lesssim\left|m_{e \tau} / m_{\mu \tau}\right| \lesssim 4.6$ & $9 \times 10^{-5} \lesssim\left|m_{e \tau}^{2} /\left(m_{e e} m_{\mu \tau}\right)\right| \lesssim 70$ \\
\hline
\end{tabular}

\begin{tabular}{|c|c|}
\hline \hline $2 \pi / 3 \leq(\delta, \beta)<4 \pi / 3$ and $0 \leq \alpha<2 \pi / 3$ \\
\hline \hline $10^{-3} \lesssim\left|m_{e e} / m_{e \mu}\right| \lesssim 7 \times 10^{2}$ & $2 \times 10^{-3} \lesssim\left|m_{e e} / m_{\mu \mu}\right| \lesssim 3 \times 10^{2}$ \\
\hline $1.3 \times 10^{-3} \lesssim\left|m_{e \tau} / m_{\mu \tau}\right| \lesssim 4.5$ & $1.5 \times 10^{-6} \lesssim\left|m_{e \tau}^{2} /\left(m_{e e} m_{\mu \tau}\right)\right| \lesssim 10^{2}$ \\
\hline
\end{tabular}

\begin{tabular}{|c|c|}
\hline \multicolumn{3}{|c|}{$2 \pi / 3 \leq \delta<4 \pi / 3,0 \leq \alpha<2 \pi / 3$ and $4 \pi / 3 \leq \beta<2 \pi$} \\
\hline \hline $9 \times 10^{-3} \lesssim\left|m_{e e} / m_{e \mu}\right| \lesssim 1.5 \times 10^{2}$ & $2 \times 10^{-3} \lesssim\left|m_{e e} / m_{\mu \mu}\right| \lesssim 2.5 \times 10^{2}$ \\
\hline $2.8 \times 10^{-3} \lesssim\left|m_{e \tau} / m_{\mu \tau}\right| \lesssim 3$ & $10^{-5} \lesssim\left|m_{e \tau}^{2} /\left(m_{e e} m_{\mu \tau}\right)\right| \lesssim 50$ \\
\hline
\end{tabular}

\begin{tabular}{|c|c|}
\hline $2 \pi / 3 \leq(\delta, \alpha)<4 \pi / 3$ and $0 \leq \beta<2 \pi / 3$ \\
\hline \hline $0.1 \lesssim\left|m_{e e} / m_{e \mu}\right| \lesssim 5 \times 10^{2}$ & $4 \times 10^{-2} \lesssim\left|m_{e e} / m_{\mu \mu}\right| \lesssim 6 \times 10^{2}$ \\
\hline $2.5 \times 10^{-3} \lesssim\left|m_{e \tau} / m_{\mu \tau}\right| \lesssim 5$ & $5 \times 10^{-6} \lesssim\left|m_{e \tau}^{2} /\left(m_{e e} m_{\mu \tau}\right)\right| \lesssim 5$ \\
\hline
\end{tabular}

\begin{tabular}{|c|c|}
\hline \multicolumn{2}{|c|}{$2 \pi / 3 \leq(\delta, \alpha, \beta)<4 \pi / 3$} \\
\hline \hline $0.1 \lesssim\left|m_{e e} / m_{e \mu}\right| \lesssim 6 \times 10^{2}$ & $3.5 \times 10^{-2} \lesssim\left|m_{e e} / m_{\mu \mu}\right| \lesssim 2.5 \times 10^{2}$ \\
\hline $2 \times 10^{-3} \lesssim\left|m_{e \tau} / m_{\mu \tau}\right| \lesssim 5$ & $5 \times 10^{-6} \lesssim\left|m_{e \tau}^{2} /\left(m_{e e} m_{\mu \tau}\right)\right| \lesssim 5$ \\
\hline
\end{tabular}

\begin{tabular}{|c|c|}
\hline \multicolumn{2}{|c|}{$2 \pi / 3 \leq(\delta, \alpha)<4 \pi / 3$ and $4 \pi / 3 \leq \beta<2 \pi$} \\
\hline \hline $0.1 \lesssim\left|m_{e e} / m_{e \mu}\right| \lesssim 6 \times 10^{2}$ & $3 \times 10^{-2} \lesssim\left|m_{e e} / m_{\mu \mu}\right| \lesssim 4 \times 10^{2}$ \\
\hline $3 \times 10^{-3} \lesssim\left|m_{e \tau} / m_{\mu \tau}\right| \lesssim 4.5$ & $10^{-5} \lesssim\left|m_{e \tau}^{2} /\left(m_{e e} m_{\mu \tau}\right)\right| \lesssim 5$ \\
\hline
\end{tabular}

\begin{tabular}{|c|c|}
\hline \multicolumn{3}{|c|}{$2 \pi / 3 \leq \delta<4 \pi / 3,4 \pi / 3 \leq \alpha<2 \pi$ and $0 \leq \beta<2 \pi / 3$} \\
\hline \hline $4 \times 10^{-3} \lesssim\left|m_{e e} / m_{e \mu}\right| \lesssim 4 \times 10^{2}$ & $4 \times 10^{-3} \lesssim\left|m_{e e} / m_{\mu \mu}\right| \lesssim 2 \times 10^{2}$ \\
\hline $2 \times 10^{-3} \lesssim\left|m_{e \tau} / m_{\mu \tau}\right| \lesssim 3$ & $3 \times 10^{-6} \lesssim\left|m_{e \tau}^{2} /\left(m_{e e} m_{\mu \tau}\right)\right| \lesssim 40$ \\
\hline
\end{tabular}

\begin{tabular}{|c|c|}
\hline \multicolumn{3}{|c|}{$2 \pi / 3 \leq(\delta, \beta)<4 \pi / 3$ and $4 \pi / 3 \leq \alpha<2 \pi$} \\
\hline \hline $7 \times 10^{-3} \lesssim\left|m_{e e} / m_{e \mu}\right| \lesssim 10^{3}$ & $2 \times 10^{-3} \lesssim\left|m_{e e} / m_{\mu \mu}\right| \lesssim 10^{2}$ \\
\hline $2 \times 10^{-3} \lesssim\left|m_{e \tau} / m_{\mu \tau}\right| \lesssim 4.5$ & $3 \times 10^{-6} \lesssim\left|m_{e \tau}^{2} /\left(m_{e e} m_{\mu \tau}\right)\right| \lesssim 30$ \\
\hline
\end{tabular}

\begin{tabular}{|c|c|}
\hline \multicolumn{3}{|c|}{$2 \pi / 3 \leq \delta<4 \pi / 3$ and $4 \pi / 3 \leq(\alpha, \beta)<2 \pi$} \\
\hline \hline $10^{-2} \lesssim\left|m_{e e} / m_{e \mu}\right| \lesssim 9 \times 10^{2}$ & $2 \times 10^{-3} \lesssim\left|m_{e e} / m_{\mu \mu}\right| \lesssim 1.5 \times 10^{2}$ \\
\hline $1.3 \times 10^{-3} \lesssim\left|m_{e \tau} / m_{\mu \tau}\right| \lesssim 4.5$ & $10^{-5} \lesssim\left|m_{e \tau}^{2} /\left(m_{e e} m_{\mu \tau}\right)\right| \lesssim 10^{2}$ \\
\hline
\end{tabular}

Table 2: Values of the renormalization invariants for the NH. 


\begin{tabular}{|c|c|}
\hline \multicolumn{2}{|c|}{$4 \pi / 3 \leq \delta<2 \pi$ and $0 \leq(\alpha, \beta)<2 \pi / 3$} \\
\hline \hline $2 \times 10^{-3} \lesssim\left|m_{e e} / m_{e \mu}\right| \lesssim 8 \times 10^{2}$ & $2.5 \times 10^{-3} \lesssim\left|m_{e e} / m_{\mu \mu}\right| \lesssim 2 \times 10^{2}$ \\
\hline $1.3 \times 10^{-3} \lesssim\left|m_{e \tau} / m_{\mu \tau}\right| \lesssim 3.2$ & $1.5 \times 10^{-5} \lesssim\left|m_{e \tau}^{2} /\left(m_{e e} m_{\mu \tau}\right)\right| \lesssim 10^{2}$ \\
\hline
\end{tabular}

\begin{tabular}{|c|c|}
\hline \hline \multicolumn{2}{|c|}{$4 \pi / 3 \leq \delta<2 \pi, 0 \leq \alpha<2 \pi / 3$ and $2 \pi / 3 \leq \beta<4 \pi / 3$} \\
\hline \hline $9 \times 10^{-3} \lesssim\left|m_{e e} / m_{e \mu}\right| \lesssim 4 \times 10^{2}$ & $3 \times 10^{-3} \lesssim\left|m_{e e} / m_{\mu \mu}\right| \lesssim 50$ \\
\hline $2.8 \times 10^{-3} \lesssim\left|m_{e \tau} / m_{\mu \tau}\right| \lesssim 3.5$ & $1.5 \times 10^{-5} \lesssim\left|m_{e \tau}^{2} /\left(m_{e e} m_{\mu \tau}\right)\right| \lesssim 60$ \\
\hline
\end{tabular}

\begin{tabular}{|c|c|}
\hline \multicolumn{3}{|c|}{$4 \pi / 3 \leq(\delta, \beta)<2 \pi$ and $0 \leq \alpha<2 \pi / 3$} \\
\hline \hline $9 \times 10^{-3} \lesssim\left|m_{e e} / m_{e \mu}\right| \lesssim 3.5 \times 10^{2}$ & $2.5 \times 10^{-3} \lesssim\left|m_{e e} / m_{\mu \mu}\right| \lesssim 5 \times 10^{2}$ \\
\hline $4 \times 10^{-3} \lesssim\left|m_{e \tau} / m_{\mu \tau}\right| \lesssim 3.5$ & $1.2 \times 10^{-4} \lesssim\left|m_{e \tau}^{2} /\left(m_{e e} m_{\mu \tau}\right)\right| \lesssim 50$ \\
\hline
\end{tabular}

\begin{tabular}{|c|c|}
\hline \multicolumn{2}{|c|}{$4 \pi / 3 \leq \delta<2 \pi, 2 \pi / 3 \leq \alpha<4 \pi / 3$ and $0 \leq \beta<2 \pi / 3$} \\
\hline \hline $0.1 \lesssim\left|m_{e e} / m_{e \mu}\right| \lesssim 4 \times 10^{2}$ & $4 \times 10^{-2} \lesssim\left|m_{e e} / m_{\mu \mu}\right| \lesssim 2 \times 10^{2}$ \\
\hline $1.5 \times 10^{-3} \lesssim\left|m_{e \tau} / m_{\mu \tau}\right| \lesssim 3.2$ & $5 \times 10^{-6} \lesssim\left|m_{e \tau}^{2} /\left(m_{e e} m_{\mu \tau}\right)\right| \lesssim 5$ \\
\hline
\end{tabular}

\begin{tabular}{|c|c|}
\hline \multicolumn{2}{|c|}{$4 \pi / 3 \leq \delta<2 \pi$ and $2 \pi / 3 \leq(\alpha, \beta)<4 \pi / 3$} \\
\hline \hline $0.1 \lesssim\left|m_{e e} / m_{e \mu}\right| \lesssim 7 \times 10^{2}$ & $3 \times 10^{-2} \lesssim\left|m_{e e} / m_{\mu \mu}\right| \lesssim 10^{2}$ \\
\hline $1.2 \times 10^{-3} \lesssim\left|m_{e \tau} / m_{\mu \tau}\right| \lesssim 5.5$ & $5 \times 10^{-6} \lesssim\left|m_{e \tau}^{2} /\left(m_{e e} m_{\mu \tau}\right)\right| \lesssim 5$ \\
\hline
\end{tabular}

\begin{tabular}{|c|c|}
\hline \multicolumn{2}{|c|}{$4 \pi / 3 \leq(\delta, \beta)<2 \pi$ and $2 \pi / 3 \leq \alpha<4 \pi / 3$} \\
\hline \hline $0.12 \lesssim\left|m_{e e} / m_{e \mu}\right| \lesssim 6 \times 10^{2}$ & $3 \times 10^{-2} \lesssim\left|m_{e e} / m_{\mu \mu}\right| \lesssim 5 \times 10^{2}$ \\
\hline $1.2 \times 10^{-3} \lesssim\left|m_{e \tau} / m_{\mu \tau}\right| \lesssim 5$ & $2 \times 10^{-6} \lesssim\left|m_{e \tau}^{2} /\left(m_{e e} m_{\mu \tau}\right)\right| \lesssim 5$ \\
\hline
\end{tabular}

\begin{tabular}{|c|c|}
\hline \multicolumn{3}{|c|}{$4 \pi / 3 \leq(\delta, \alpha)<2 \pi$ and $0 \leq \beta<2 \pi / 3$} \\
\hline \hline $5 \times 10^{-3} \lesssim\left|m_{e e} / m_{e \mu}\right| \lesssim 4 \times 10^{2}$ & $2 \times 10^{-3} \lesssim\left|m_{e e} / m_{\mu \mu}\right| \lesssim 1.5 \times 10^{2}$ \\
\hline $1.2 \times 10^{-3} \lesssim\left|m_{e \tau} / m_{\mu \tau}\right| \lesssim 3.5$ & $3 \times 10^{-6} \lesssim\left|m_{e \tau}^{2} /\left(m_{e e} m_{\mu \tau}\right)\right| \lesssim 30$ \\
\hline
\end{tabular}

\begin{tabular}{|c|c|}
\hline \multicolumn{3}{|c|}{$4 \pi / 3 \leq(\delta, \alpha)<2 \pi$ and $2 \pi / 3 \leq \beta<4 \pi / 3$} \\
\hline \hline $6 \times 10^{-3} \lesssim\left|m_{e e} / m_{e \mu}\right| \lesssim 6 \times 10^{2}$ & $2.5 \times 10^{-3} \lesssim\left|m_{e e} / m_{\mu \mu}\right| \lesssim 10^{2}$ \\
\hline $2 \times 10^{-3} \lesssim\left|m_{e \tau} / m_{\mu \tau}\right| \lesssim 4.5$ & $3 \times 10^{-6} \lesssim\left|m_{e \tau}^{2} /\left(m_{e e} m_{\mu \tau}\right)\right| \lesssim 40$ \\
\hline
\end{tabular}

Table 3: Values of the renormalization invariants for the NH. 


\begin{tabular}{|c|c|}
\hline \multicolumn{2}{|c|}{$0 \leq(\delta, \alpha)<2 \pi / 3$ and $2 \pi / 3 \leq \beta<4 \pi / 3$} \\
\hline \hline $0.3 \lesssim\left|m_{e e} / m_{e \mu}\right| \lesssim 5 \times 10^{2}$ & $0.3 \lesssim\left|m_{e e} / m_{\mu \mu}\right| \lesssim 7 \times 10^{2}$ \\
\hline $1.8 \times 10^{-3} \lesssim\left|m_{e \tau} / m_{\mu \tau}\right| \lesssim 6.5$ & $1.5 \times 10^{-6} \lesssim\left|m_{e \tau}^{2} /\left(m_{e e} m_{\mu \tau}\right)\right| \lesssim 3$ \\
\hline
\end{tabular}

\begin{tabular}{|c|c|}
\hline \multicolumn{3}{|c|}{$0 \leq(\delta, \alpha)<2 \pi / 3$ and $4 \pi / 3 \leq \beta<2 \pi$} \\
\hline \hline $0.3 \lesssim\left|m_{e e} / m_{e \mu}\right| \lesssim 7 \times 10^{2}$ & $0.3 \lesssim\left|m_{e e} / m_{\mu \mu}\right| \lesssim 4 \times 10^{2}$ \\
\hline $1.8 \times 10^{-3} \lesssim\left|m_{e \tau} / m_{\mu \tau}\right| \lesssim 3.2$ & $2 \times 10^{-6} \lesssim\left|m_{e \tau}^{2} /\left(m_{e e} m_{\mu \tau}\right)\right| \lesssim 0.6$ \\
\hline
\end{tabular}

\begin{tabular}{|c|c|}
\hline \multicolumn{3}{|c|}{$0 \leq(\delta, \beta)<2 \pi / 3$ and $2 \pi / 3 \leq \alpha<4 \pi / 3$} \\
\hline \hline $0.7 \lesssim\left|m_{e e} / m_{e \mu}\right| \lesssim 1.2 \times 10^{3}$ & $0.5 \lesssim\left|m_{e e} / m_{\mu \mu}\right| \lesssim 4 \times 10^{2}$ \\
\hline $3 \times 10^{-3} \lesssim\left|m_{e \tau} / m_{\mu \tau}\right| \lesssim 8$ & $8 \times 10^{-6} \lesssim\left|m_{e \tau}^{2} /\left(m_{e e} m_{\mu \tau}\right)\right| \lesssim 5$ \\
\hline
\end{tabular}

\begin{tabular}{|c|c|}
\hline \multicolumn{3}{|c|}{$0 \leq \delta<2 \pi / 3$ and $2 \pi / 3 \leq(\alpha, \beta)<4 \pi / 3$} \\
\hline \hline $0.7 \lesssim\left|m_{e e} / m_{e \mu}\right| \lesssim 1.7 \times 10^{3}$ & $0.5 \lesssim\left|m_{e e} / m_{\mu \mu}\right| \lesssim 3 \times 10^{2}$ \\
\hline $1.2 \times 10^{-3} \lesssim\left|m_{e \tau} / m_{\mu \tau}\right| \lesssim 8$ & $8 \times 10^{-7} \lesssim\left|m_{e \tau}^{2} /\left(m_{e e} m_{\mu \tau}\right)\right| \lesssim 5$ \\
\hline
\end{tabular}

\begin{tabular}{|c|c|}
\hline \multicolumn{2}{|c|}{$0 \leq \delta<2 \pi / 3,2 \pi / 3 \leq \alpha<4 \pi / 3$ and $4 \pi / 3 \leq \beta<2 \pi$} \\
\hline \hline $0.7 \lesssim\left|m_{e e} / m_{e \mu}\right| \lesssim 5 \times 10^{2}$ & $0.5 \lesssim\left|m_{e e} / m_{\mu \mu}\right| \lesssim 4 \times 10^{2}$ \\
\hline $1.2 \times 10^{-3} \lesssim\left|m_{e \tau} / m_{\mu \tau}\right| \lesssim 4$ & $1.8 \times 10^{-6} \lesssim\left|m_{e \tau}^{2} /\left(m_{e e} m_{\mu \tau}\right)\right| \lesssim 4$ \\
\hline
\end{tabular}

\begin{tabular}{|c|c|}
\hline \multicolumn{2}{|c|}{$0 \leq(\delta, \beta)<2 \pi / 3$ and $4 \pi / 3 \leq \alpha<2 \pi$} \\
\hline \hline $0.2 \lesssim\left|m_{e e} / m_{e \mu}\right| \lesssim 8 \times 10^{2}$ & $0.3 \lesssim\left|m_{e e} / m_{\mu \mu}\right| \lesssim 8 \times 10^{2}$ \\
\hline $2 \times 10^{-2} \lesssim\left|m_{e \tau} / m_{\mu \tau}\right| \lesssim 4.1$ & $4 \times 10^{-4} \lesssim\left|m_{e \tau}^{2} /\left(m_{e e} m_{\mu \tau}\right)\right| \lesssim 6$ \\
\hline
\end{tabular}

\begin{tabular}{|c|c|}
\hline \multicolumn{2}{|c|}{$0 \leq \delta<2 \pi / 3,4 \pi / 3 \leq \alpha<2 \pi$ and $2 \pi / 3 \leq \beta<4 \pi / 3$} \\
\hline \hline $0.3 \lesssim\left|m_{e e} / m_{e \mu}\right| \lesssim 8 \times 10^{2}$ & $0.3 \lesssim\left|m_{e e} / m_{\mu \mu}\right| \lesssim 2.2 \times 10^{2}$ \\
\hline $4 \times 10^{-3} \lesssim\left|m_{e \tau} / m_{\mu \tau}\right| \lesssim 5$ & $2 \times 10^{-6} \lesssim\left|m_{e \tau}^{2} /\left(m_{e e} m_{\mu \tau}\right)\right| \lesssim 2$ \\
\hline
\end{tabular}

\begin{tabular}{|c|c|}
\hline \multicolumn{2}{|c|}{$0 \leq \delta<2 \pi / 3$ and $4 \pi / 3 \leq(\alpha, \beta)<2 \pi$} \\
\hline \hline $0.3 \lesssim\left|m_{e e} / m_{e \mu}\right| \lesssim 5 \times 10^{2}$ & $0.3 \lesssim\left|m_{e e} / m_{\mu \mu}\right| \lesssim 2 \times 10^{2}$ \\
\hline $9 \times 10^{-3} \lesssim\left|m_{e \tau} / m_{\mu \tau}\right| \lesssim 4$ & $2 \times 10^{-5} \lesssim\left|m_{e \tau}^{2} /\left(m_{e e} m_{\mu \tau}\right)\right| \lesssim 6$ \\
\hline
\end{tabular}

Table 4: Values of the renormalization invariants for the IH. 


\begin{tabular}{|c|c|}
\hline \multicolumn{2}{|c|}{$2 \pi / 3 \leq \delta<4 \pi / 3$ and $0 \leq(\alpha, \beta)<2 \pi / 3$} \\
\hline \hline $0.4 \lesssim\left|m_{e e} / m_{e \mu}\right| \lesssim 1.8 \times 10^{3}$ & $0.3 \lesssim\left|m_{e e} / m_{\mu \mu}\right| \lesssim 2 \times 10^{2}$ \\
\hline $7 \times 10^{-3} \lesssim\left|m_{e \tau} / m_{\mu \tau}\right| \lesssim 5.5$ & $5 \times 10^{-5} \lesssim\left|m_{e \tau}^{2} /\left(m_{e e} m_{\mu \tau}\right)\right| \lesssim 9$ \\
\hline
\end{tabular}

\begin{tabular}{|c|c|}
\hline \hline \multicolumn{2}{|c|}{$2 \pi / 3 \leq(\delta, \beta)<4 \pi / 3$ and $0 \leq \alpha<2 \pi / 3$} \\
\hline \hline $0.4 \lesssim\left|m_{e e} / m_{e \mu}\right| \lesssim 8 \times 10^{2}$ & $0.3 \lesssim\left|m_{e e} / m_{\mu \mu}\right| \lesssim 1.2 \times 10^{2}$ \\
\hline $2 \times 10^{-3} \lesssim\left|m_{e \tau} / m_{\mu \tau}\right| \lesssim 6$ & $1.5 \times 10^{-6} \lesssim\left|m_{e \tau}^{2} /\left(m_{e e} m_{\mu \tau}\right)\right| \lesssim 5$ \\
\hline
\end{tabular}

\begin{tabular}{|c|c|}
\hline \multicolumn{2}{|c|}{$2 \pi / 3 \leq \delta<4 \pi / 3,0 \leq \alpha<2 \pi / 3$ and $4 \pi / 3 \leq \beta<2 \pi$} \\
\hline \hline $0.4 \lesssim\left|m_{e e} / m_{e \mu}\right| \lesssim 5 \times 10^{2}$ & $0.3 \lesssim\left|m_{e e} / m_{\mu \mu}\right| \lesssim 2 \times 10^{2}$ \\
\hline $2 \times 10^{-3} \lesssim\left|m_{e \tau} / m_{\mu \tau}\right| \lesssim 5.5$ & $2 \times 10^{-6} \lesssim\left|m_{e \tau}^{2} /\left(m_{e e} m_{\mu \tau}\right)\right| \lesssim 5$ \\
\hline
\end{tabular}

\begin{tabular}{|c|c|}
\hline \multicolumn{3}{|c|}{$2 \pi / 3 \leq(\delta, \alpha)<4 \pi / 3$ and $0 \leq \beta<2 \pi / 3$} \\
\hline \hline $0.7 \lesssim\left|m_{e e} / m_{e \mu}\right| \lesssim 4 \times 10^{2}$ & $0.5 \lesssim\left|m_{e e} / m_{\mu \mu}\right| \lesssim 2 \times 10^{2}$ \\
\hline $1.8 \times 10^{-3} \lesssim\left|m_{e \tau} / m_{\mu \tau}\right| \lesssim 6$ & $1.1 \times 10^{-6} \lesssim\left|m_{e \tau}^{2} /\left(m_{e e} m_{\mu \tau}\right)\right| \lesssim 5$ \\
\hline
\end{tabular}

\begin{tabular}{|c|c|}
\hline \multicolumn{2}{|c|}{$2 \pi / 3 \leq(\delta, \alpha, \beta)<4 \pi / 3$} \\
\hline \hline $0.7 \lesssim\left|m_{e e} / m_{e \mu}\right| \lesssim 8 \times 10^{2}$ & $0.5 \lesssim\left|m_{e e} / m_{\mu \mu}\right| \lesssim 1.8 \times 10^{2}$ \\
\hline $1.8 \times 10^{-3} \lesssim\left|m_{e \tau} / m_{\mu \tau}\right| \lesssim 6.5$ & $1.3 \times 10^{-6} \lesssim\left|m_{e \tau}^{2} /\left(m_{e e} m_{\mu \tau}\right)\right| \lesssim 5$ \\
\hline
\end{tabular}

\begin{tabular}{|c|c|}
\hline $2 \pi / 3 \leq(\delta, \alpha)<4 \pi / 3$ and $4 \pi / 3 \leq \beta<2 \pi$ \\
\hline \hline $0.7 \lesssim\left|m_{e e} / m_{e \mu}\right| \lesssim 3 \times 10^{3}$ & $0.5 \lesssim\left|m_{e e} / m_{\mu \mu}\right| \lesssim 5 \times 10^{2}$ \\
\hline $3.2 \times 10^{-3} \lesssim\left|m_{e \tau} / m_{\mu \tau}\right| \lesssim 5.2$ & $10^{-5} \lesssim\left|m_{e \tau}^{2} /\left(m_{e e} m_{\mu \tau}\right)\right| \lesssim 5$ \\
\hline
\end{tabular}

\begin{tabular}{|c|c|}
\hline \multicolumn{2}{|c|}{$2 \pi / 3 \leq \delta<4 \pi / 3,4 \pi / 3 \leq \alpha<2 \pi$ and $0 \leq \beta<2 \pi / 3$} \\
\hline \hline $0.4 \lesssim\left|m_{e e} / m_{e \mu}\right| \lesssim 3 \times 10^{2}$ & $0.3 \lesssim\left|m_{e e} / m_{\mu \mu}\right| \lesssim 2 \times 10^{2}$ \\
\hline $3 \times 10^{-3} \lesssim\left|m_{e \tau} / m_{\mu \tau}\right| \lesssim 4$ & $10^{-5} \lesssim\left|m_{e \tau}^{2} /\left(m_{e e} m_{\mu \tau}\right)\right| \lesssim 5$ \\
\hline
\end{tabular}

\begin{tabular}{|c|c|}
\hline $2 \pi / 3 \leq(\delta, \beta)<4 \pi / 3$ and $4 \pi / 3 \leq \alpha<2 \pi$ \\
\hline \hline $0.4 \lesssim\left|m_{e e} / m_{e \mu}\right| \lesssim 8 \times 10^{2}$ & $0.3 \lesssim\left|m_{e e} / m_{\mu \mu}\right| \lesssim 3 \times 10^{2}$ \\
\hline $2 \times 10^{-3} \lesssim\left|m_{e \tau} / m_{\mu \tau}\right| \lesssim 5.5$ & $10^{-6} \lesssim\left|m_{e \tau}^{2} /\left(m_{e e} m_{\mu \tau}\right)\right| \lesssim 5$ \\
\hline
\end{tabular}

\begin{tabular}{|c|c|}
\hline \multicolumn{3}{|c|}{$2 \pi / 3 \leq \delta<4 \pi / 3$ and $4 \pi / 3 \leq(\alpha, \beta)<2 \pi$} \\
\hline \hline $0.4 \lesssim\left|m_{e e} / m_{e \mu}\right| \lesssim 8 \times 10^{2}$ & $0.2 \lesssim\left|m_{e e} / m_{\mu \mu}\right| \lesssim 5 \times 10^{2}$ \\
\hline $2 \times 10^{-3} \lesssim\left|m_{e \tau} / m_{\mu \tau}\right| \lesssim 5.5$ & $2 \times 10^{-6} \lesssim\left|m_{e \tau}^{2} /\left(m_{e e} m_{\mu \tau}\right)\right| \lesssim 5$ \\
\hline
\end{tabular}

Table 5: Values of the renormalization invariants for the IH. 


\begin{tabular}{|c|c|}
\hline \multicolumn{2}{|c|}{$4 \pi / 3 \leq \delta<2 \pi$ and $0 \leq(\alpha, \beta)<2 \pi / 3$} \\
\hline \hline $0.3 \lesssim\left|m_{e e} / m_{e \mu}\right| \lesssim 5 \times 10^{2}$ & $0.3 \lesssim\left|m_{e e} / m_{\mu \mu}\right| \lesssim 4 \times 10^{2}$ \\
\hline $10^{-3} \lesssim\left|m_{e \tau} / m_{\mu \tau}\right| \lesssim 4$ & $1.8 \times 10^{-5} \lesssim\left|m_{e \tau}^{2} /\left(m_{e e} m_{\mu \tau}\right)\right| \lesssim 5$ \\
\hline
\end{tabular}

\begin{tabular}{|c|c|}
\hline \hline \multicolumn{2}{|c|}{$4 \pi / 3 \leq \delta<2 \pi, 0 \leq \alpha<2 \pi / 3$ and $2 \pi / 3 \leq \beta<4 \pi / 3$} \\
\hline \hline $0.3 \lesssim\left|m_{e e} / m_{e \mu}\right| \lesssim 9 \times 10^{2}$ & $0.3 \lesssim\left|m_{e e} / m_{\mu \mu}\right| \lesssim 70$ \\
\hline $4 \times 10^{-3} \lesssim\left|m_{e \tau} / m_{\mu \tau}\right| \lesssim 4$ & $8 \times 10^{-6} \lesssim\left|m_{e \tau}^{2} /\left(m_{e e} m_{\mu \tau}\right)\right| \lesssim 5$ \\
\hline
\end{tabular}

\begin{tabular}{|c|c|}
\hline \multicolumn{3}{|c|}{$4 \pi / 3 \leq(\delta, \beta)<2 \pi$ and $0 \leq \alpha<2 \pi / 3$} \\
\hline \hline $0.3 \lesssim\left|m_{e e} / m_{e \mu}\right| \lesssim 3.5 \times 10^{2}$ & $0.3 \lesssim\left|m_{e e} / m_{\mu \mu}\right| \lesssim 6 \times 10^{2}$ \\
\hline $2.2 \times 10^{-2} \lesssim\left|m_{e \tau} / m_{\mu \tau}\right| \lesssim 4$ & $2 \times 10^{-4} \lesssim\left|m_{e \tau}^{2} /\left(m_{e e} m_{\mu \tau}\right)\right| \lesssim 5$ \\
\hline
\end{tabular}

\begin{tabular}{|c|c|}
\hline \multicolumn{2}{|c|}{$4 \pi / 3 \leq \delta<2 \pi, 2 \pi / 3 \leq \alpha<4 \pi / 3$ and $0 \leq \beta<2 \pi / 3$} \\
\hline \hline $0.6 \lesssim\left|m_{e e} / m_{e \mu}\right| \lesssim 6 \times 10^{2}$ & $0.5 \lesssim\left|m_{e e} / m_{\mu \mu}\right| \lesssim 5 \times 10^{2}$ \\
\hline $1.2 \times 10^{-3} \lesssim\left|m_{e \tau} / m_{\mu \tau}\right| \lesssim 4$ & $1.2 \times 10^{-6} \lesssim\left|m_{e \tau}^{2} /\left(m_{e e} m_{\mu \tau}\right)\right| \lesssim 5$ \\
\hline
\end{tabular}

\begin{tabular}{|c|c|}
\hline \multicolumn{2}{|c|}{$4 \pi / 3 \leq \delta<2 \pi$ and $2 \pi / 3 \leq(\alpha, \beta)<4 \pi / 3$} \\
\hline \hline $0.6 \lesssim\left|m_{e e} / m_{e \mu}\right| \lesssim 7 \times 10^{2}$ & $0.5 \lesssim\left|m_{e e} / m_{\mu \mu}\right| \lesssim 10^{2}$ \\
\hline $3 \times 10^{-3} \lesssim\left|m_{e \tau} / m_{\mu \tau}\right| \lesssim 6$ & $1.2 \times 10^{-6} \lesssim\left|m_{e \tau}^{2} /\left(m_{e e} m_{\mu \tau}\right)\right| \lesssim 5$ \\
\hline
\end{tabular}

\begin{tabular}{|c|c|}
\hline \multicolumn{2}{|c|}{$4 \pi / 3 \leq(\delta, \beta)<2 \pi$ and $2 \pi / 3 \leq \alpha<4 \pi / 3$} \\
\hline \hline $0.6 \lesssim\left|m_{e e} / m_{e \mu}\right| \lesssim 8 \times 10^{2}$ & $0.4 \lesssim\left|m_{e e} / m_{\mu \mu}\right| \lesssim 2 \times 10^{2}$ \\
\hline $1.2 \times 10^{-3} \lesssim\left|m_{e \tau} / m_{\mu \tau}\right| \lesssim 7.5$ & $10^{-6} \lesssim\left|m_{e \tau}^{2} /\left(m_{e e} m_{\mu \tau}\right)\right| \lesssim 5$ \\
\hline
\end{tabular}

\begin{tabular}{|c|c|}
\hline \multicolumn{2}{|c|}{$4 \pi / 3 \leq(\delta, \alpha)<2 \pi$ and $0 \leq \beta<2 \pi / 3$} \\
\hline \hline $0.2 \lesssim\left|m_{e e} / m_{e \mu}\right| \lesssim 3.5 \times 10^{2}$ & $0.2 \lesssim\left|m_{e e} / m_{\mu \mu}\right| \lesssim 3 \times 10^{2}$ \\
\hline $3 \times 10^{-3} \lesssim\left|m_{e \tau} / m_{\mu \tau}\right| \lesssim 4$ & $10^{-5} \lesssim\left|m_{e \tau}^{2} /\left(m_{e e} m_{\mu \tau}\right)\right| \lesssim 5$ \\
\hline
\end{tabular}

\begin{tabular}{|c|c|}
\hline \multicolumn{2}{|c|}{$4 \pi / 3 \leq(\delta, \alpha)<2 \pi$ and $2 \pi / 3 \leq \beta<4 \pi / 3$} \\
\hline \hline $0.3 \lesssim\left|m_{e e} / m_{e \mu}\right| \lesssim 10^{3}$ & $0.2 \lesssim\left|m_{e e} / m_{\mu \mu}\right| \lesssim 5 \times 10^{2}$ \\
\hline $3 \times 10^{-3} \lesssim\left|m_{e \tau} / m_{\mu \tau}\right| \lesssim 6$ & $3 \times 10^{-6} \lesssim\left|m_{e \tau}^{2} /\left(m_{e e} m_{\mu \tau}\right)\right| \lesssim 5$ \\
\hline
\end{tabular}

Table 6: Values of the renormalization invariants for the IH. 
[6] P. H. Chankowski and Z. Pluciennik, Phys. Lett. B 316 (1993) 312 hep-ph/9306333; K. S. Babu, C. N. Leung and J. T. Pantaleone, Phys. Lett. B 319 (1993) 191 hep-ph/9309223.

[7] J. R. Ellis and S. Lola, Phys. Lett. B 458 (1999) 310 hep-ph/9904279].

[8] N. Haba, N. Okamura and M. Sugiura, Prog. Theor. Phys. 103 (2000) 367 hep-ph/9810471; N. Haba, Y. Matsui, N. Okamura and M. Sugiura, Prog. Theor. Phys. 103 (2000) 145 [hep-ph/9908429]; N. Haba, Y. Matsui and N. Okamura, Prog. Theor. Phys. 103 (2000) 807 hep-ph/9911481; Eur. Phys. J. C 17 (2000) 513 hep-ph/0005075]; N. Haba, Y. Matsui, N. Okamura and T. Suzuki, Phys. Lett. B 489 (2000) 184 [hep-ph/0005064]; S. Antusch, J. Kersten, M. Lindner and M. Ratz, Nucl. Phys. B 674 (2003) 401 hep-ph/0305273]; S. Antusch, J. Kersten, M. Lindner, M. Ratz and M. A. Schmidt, JHEP 0503 (2005) 024 hep-ph/0501272; J. -w. Mei, Phys. Rev. D 71 (2005) 073012 J. -w. Mei and Z. -z. Xing, Phys. Lett. B 623 (2005) 227 hep-ph/0506304; S. Luo, J. -w. Mei and Z. -z. Xing, Phys. Rev. D 72 (2005) 053014 hep-ph/0507065); S. Ray, W. Rodejohann and M. A. Schmidt, Phys. Rev. D 83 (2011) 033002 [arXiv:1010.1206 [hep-ph]]; S. Luo and Z. -z. Xing, arXiv:1203.3118 [hep-ph].

[9] N. Haba, Y. Matsui, N. Okamura and M. Sugiura, Eur. Phys. J. C 10 (1999) 677 hep-ph/9904292.

[10] N. Haba and N. Okamura, Eur. Phys. J. C 14 (2000) 347 [hep-ph/9906481].

[11] N. Haba and R. Takahashi, Europhys. Lett. 100 (2012) 31001 arXiv:1206.2793 [hepph]]; N. Haba, K. Kaneta and R. Takahashi, Europhys. Lett. 101 (2013) 11001 arXiv:1209.1522 [hep-ph]].

[12] S. Weinberg, Phys. Rev. Lett. 43 (1979) 1566.

[13] R. N. Mohapatra and W. Rodejohann, Phys. Lett. B 644 (2007) 59 hep-ph/0608111.

[14] P. A. R. Ade et al. [Planck Collaboration], arXiv:1303.5076 [astro-ph.CO]; C. L. Bennett, D. Larson, J. L. Weiland, N. Jarosik, G. Hinshaw, N. Odegard, K. M. Smith and R. S. Hill et al., arXiv:1212.5225 [astro-ph.CO]; P. A. R. Ade et al. [Planck Collaboration], arXiv:1303.5075 [astro-ph.CO].

[15] N. Haba and R. Takahashi, arXiv:1305.0147 [hep-ph]. 